\title{
Assessment of Postoperative Outcomes in Spinal Epidural Abscess Following Surgical Decompression
}

Leonard J. Keller, B.S. ${ }^{1,2}$; Vincent J. Alentado, M.D. ${ }^{3}$; Joseph E. Tanenbaum, B.A. ${ }^{1,2,7}$;Bryan S. Lee, M.D. ${ }^{1,4,5}$; Amy S. Nowacki, PhD ${ }^{5,8}$; Edward C. Benzel, M.D. ${ }^{1,4,5}$; Thomas E. Mroz, M.D. ${ }^{1,4,6}$ Michael P. Steinmetz, M.D. ${ }^{1,4,5^{*}}$

enter for Spine Health, Cleveland Clinic, 9500 Euclid Avenue, Cleveland, Ohio, 44195, USA

${ }^{2}$ Case Western Reserve University School of Medicine, 10900 Euclid Avenue, Cleveland, Ohio, 44106, USA

${ }^{3}$ Department of Neurological Surgery, Indiana University School of Medicine, 355 W. 16th

Street, Goodman Hall Suite 5100, Indianapolis, IN 46202, USA

${ }^{4}$ Department of Neurosurgery, Cleveland Clinic, 9500 Euclid Avenue, Cleveland, Ohio, 44195, USA

${ }^{5}$ Cleveland Clinic Lerner College of Medicine, Cleveland Clinic, 9500 Euclid Avenue, Cleveland, Ohio, 44195, USA

${ }^{6}$ Department of Orthopaedic Surgery, Cleveland Clinic, 9500 Euclid Avenue, Cleveland, Ohio, 44195, USA

${ }^{7}$ Department of Epidemiology and Biostatistics, Case Western Reserve University, 10900 Euclid Avenue, Cleveland, Ohio, 44106, USA

${ }^{8}$ Department of Quantitative Health Sciences, Cleveland Clinic, 9500 Euclid Avenue, Cleveland, Ohio, 44195, USA

Email addresses of authors: Leonard J. Keller(1jk54@case.edu); Vincent J. Alentado (valentado@gmail.com); Joseph E. Tanenbaum (jet94@case.edu); Bryan S. Lee (bryansukbae.lee@gmail.com); Amy S. Nowacki (nowacka@ccf.org); Edward C. Benzel (benzele@ccf.org); Thomas E. Mroz (mrozt@ccf.org); Michael P. Steinmetz (steinmm@ccf.org)

This is the author's manuscript of the article published in final edited form as:

Keller, L. J., Alentado, V. J., Tanenbaum, J. E., Lee, B. S., Nowacki, A. S., Benzel, E. C., ... Steinmetz, M. P. (2018). Assessment of Postoperative Outcomes in Spinal Epidural Abscess Following Surgical Decompression. The Spine Journal. https://doi.org/10.1016/j.spinee.2018.12.006 
* Corresponding Author:

Leonard J. Keller

Center for Spine Health

Department of Neurosurgery

Neurological Institute

The Cleveland Clinic

9500 Euclid Avenue, S-80

Cleveland, Ohio 44195

Tel: (216) 444-2200

Email:1jk54@case.edu

\section{Abstract}

Background Context: A spinal epidural abscess (SEA) is a serious condition that may be managed with antibiotics alone or with decompressive surgery combined with antibiotics.

Purpose: The objectives of this study were to assess the clinical outcomes of SEA after surgical management and to identify the patient-level factors that are associated with outcomes following surgical decompression and removal of SEA.

Study Design/Setting. Retrospective chart review analysis.

Patient Sample: An analysis of 154 consecutive patients who initially presented to a tertiarycare, academic medical center with SEA, and were subsequently treated with surgery between 2010 and 2015 was performed.

Outcome Measures: Postoperative pre-discharge American Spinal Injury Association Impairment Scale (AIS) scores, 6-month follow-up encounter AIS scores, need for revision surgery, and mortality during SEA surgery were the primary outcomes.

Physiologic Measures: AIS scores 
Method: Fisher's exact and Wilcoxon rank-sum tests were used to assess the associations between patient-level factors and surgical outcomes. Moreover, an interactive, predictive model for postoperative pre-discharge AIS score was developed using a proportional odds regression model. There was no funding secured for this study and there are no conflict of interestassociated biases.

Results: 154 patients (mean age of 58 years) were treated using surgical decompression in addition to antibiotics. The majority of patients were Caucasian (81\%) and male (61\%). No intraoperative mortality was reported. A second SEA surgery was performed in $8 \%$ of patients. A comparison of the preoperative and postoperative pre-discharge AIS scores showed that $49 \%$ of patients maintained a score of $\mathrm{E}$ or improved, while $45 \%$ remained at their preoperative status and $6 \%$ worsened. Among a subset of patients $(n=36 ; 23 \%)$ for whom a 6 month follow-up encounter occurred, $75 \%$ maintained an AIS score of E or improved, $19 \%$ remained at their preoperative status, and $6 \%$ worsened. Both the presence and longer duration of preoperative paresis was associated with an increased risk of remaining at the same AIS score or worsening at the predischarge encounter (both $\mathrm{p}<0.001$ ). A predictive model for predischarge AIS scores was developed based on several patient characteristics.

Conclusions: Surgical decompression can contribute to improving or maintaining AIS scores in a high percentage of SEA patients. The presence and duration of preoperative paresis are prognostic for poorer outcomes and suggest that rapid surgical intervention before paresis develops may lead to improved postoperative outcomes. Our modeling tool enables an estimation of probabilities of patients' predischarge condition.

Keywords; spinal epidural abscess; infection; surgery; paresis; American Spinal Injury Association Impairment Scale 


\section{Introduction}

Spinal epidural abscess (SEA) is a pyogenic infection located between the dura mater and the bony confines of the spine. ${ }^{1}$ While SEA is a rare occurrence, the potential for serious morbidity and mortality is high, with an estimated mortality rate ranging from $1.8-25 \%$ and $45 \%$ of patients suffer from long-term neurologic dysfunction. ${ }^{2,3,4}$ When treated operatively, the most common approach is posterior laminectomy with washout of the epidural space. ${ }^{5}$ Though SEA has a high potential for morbidity and mortality, to date there are no comprehensive evidence-based criteria for predicting the outcomes after treating SEA via operative management. Several prior studies analyzed the risk factors for poor outcomes in patients with SEA, although many of these studies focused on medical and surgical management, and no study used a multivariable model that included all of the risk factors linked to poor perioperative outcomes specifically. , $2,4,6-8^{-}$

Given the limited number of studies focused on surgical management of SEA, evidence-based guidance for managing such patients would prove useful. Therefore, this study sought to characterize the clinical outcomes and to identify the patient-level factors that are associated with such outcomes following surgical decompression and removal of SEA. Additionally, we attempted to develop an interactive, predictive model for postoperative, predischarge AIS scores.

\section{Methods}

\section{Overview and Study Design}

After obtaining internal review board approval, a retrospective analysis of 154 consecutive patients that presented to a tertiary-care, academic medical center with SEA and that were treated with any surgery between 2010 and 2015 was performed. Electronic medical records were accessed to gather patient characteristics, imaging studies, and pre/post-surgical neurologic status.

Severity of thecal sac compression was classified into two categories. If spinal canal involvement on the axial view of the magnetic resonance image (MRI) or computed tomography (CT) was less than $50 \%$, the compression was judged to be mild to moderate. If the spinal canal 
involvement was $50 \%$ or greater, then the compression was judged to be severe. MRI was used if both MRI and CT were available. This approach is the same as the study by Khanna et al. ${ }^{4}$

\section{Study Population}

Any patient younger than 18 years of age was excluded on the basis of skeletal immaturity. Additionally, any patients who had spinal malignancy, neuromuscular disease, or Pott's disease were excluded in order to eliminate the confounding effect of these pathologies.

\section{Outcomes}

The outcomes investigated were postoperative, predischarge American Spinal Injury Association Impairment Scale (AIS) ${ }^{8}$ scores, 6 month ( \pm 2 month) follow-up encounter AIS scores, death during SEA surgery, and reoperation. AIS scores assess the extent of the spinal injury and can range from a value of $\mathrm{A}$ (Complete) to $\mathrm{E}$ (neurologically intact). Of note, both the achievement of good status (levels E/D) and status improvement can be viewed as successful surgical outcomes. Therefore, AIS scores were categorized according to both status achievement (levels $\mathrm{E} / \mathrm{D}$ vs. levels $\mathrm{C} / \mathrm{B} / \mathrm{A}$ ) and status change (improvement vs. remaining the same/worsening with respect to preoperative AIS score).

\section{Analytic Approach}

SEA surgical patient and abscess characteristics are summarized with appropriate descriptive statistics (count $(\%)$, mean \pm standard deviation, or median $\left(\mathrm{Q}_{1}-\mathrm{Q}_{3}\right)$ ). Fisher's exact and Wilcoxon rank-sum tests were used to assess the associations between both patient-level and abscess-level factors and the categorical version of the AIS outcomes. A prediction model was developed to predict the ordinal outcome of postoperative, predischarge AIS score according to preoperative patient factors using a multivariable proportional odds regression model. The proportional odds assumption was verified with a score test and a leave-one-out cross-validation was performed. An alpha level of 0.05 was used to determine statistical significance. Analyses 
were conducted with SAS version 9.4 (SAS Institute, Cary, NC) and R version 3.3.1 (R Core Team, Vienna, Austria) statistical software.

\section{Results}

\section{Patient Population}

A total of 154 patients (mean age, 58 years) who met the inclusion criteria as described above, and their complete demographic data were obtained (Tables 1 and 2). The majority of patients were Caucasian (81\%) and male (61\%). Age, race, and sex were not found to be associated with predischarge AIS scores ( $\mathrm{p}=0.61,0.30$, and 0.87 , respectively). See Tables 1 and 2 .

\section{Patient Characteristics Associated with Predischarge AIS Scores}

The presence of preoperative paresis was associated with remaining at the same or a worsening AIS score at the postoperative predischarge encounter $(\mathrm{p}<0.001)$. An increasing duration of preoperative paresis (mean value of 1 day) was also associated with remaining at the same or a worsening AIS score at the predischarge encounter $(\mathrm{p}<0.001)$. Other patient characteristics were not associated with unchanged or worsening AIS score at the predischarge encounter compared to their preoperative state, though some were relevant in the AIS score prediction model. See Table 2.

The SEA's were found to be located more in the lumbar (28\%) and thoracic regions (27\%) than in the cervical region (21\%) with some SEA's spanning multiple regions. Dorsal SEA's accounted for $41 \%$ of the cases and $36 \%$ were located ventrally. Those classified as 'other" were indeterminate. Most cases (58\%) were categorized as severe thecal sac compression and 34\% were mild to moderate. Three of the images were unable to be read due to image quality and for 10 patients there was no MRI/CT available. See Table 3. There was no association found between the region of the abscess, the location of the abscess, or the degree of thecal sac 
compression and the pre-discharge evaluation ( $\mathrm{p}=0.17,0.13$, and 0.39 , respectively). See Table 4.

\section{Patient Characteristics Associated with Follow-up AIS Scores}

In the subset of patients $(\mathrm{n}=36 ; 23 \%)$ for whom a 6 month follow-up evaluation was available, the presence of paraspinal abscess, increased preoperative C-reactive protein (CRP), and increased preoperative platelets were associated with an increased risk of remaining at the same or worsening AIS score at the 6 month follow-up ( $p=0.02,0.049$, and 0.049 , respectively). See Table 2. The mean time to the post-discharge encounter was 5.7 months. A significant association was found between degree of thecal sac compression and remaining at the same or worsening AIS score at the 6 month encounter $(p=0.007)$. See Table 4.

\section{Pre-discharge and Follow-up AIS Scores}

At the pre-discharge evaluation (mean time from surgery to evaluation 7 days), $79 \%$ of patients achieved an E or D AIS score while $21 \%$ achieved an A, B, or C score. Among a subset of patients $(\mathrm{n}=36 ; 23 \%)$ for whom a 6 month follow-up encounter occurred, $97 \%$ achieved an $\mathrm{E}$ or D AIS score while 3\% achieved an A, B, or C score. See Table 5.

A comparison of the preoperative AIS and postoperative pre-discharge AIS scores showed that $49 \%$ of patients maintained an AIS score of E or improved while $45 \%$ remained at their preoperative status and 6\% worsened. Among a subset of patients $(n=36)$ for whom a 6 month follow-up encounter occurred, 75\% maintained an AIS score of E or improved, 19\% remained at their preoperative status, and 6\% worsened. This is summarized in Figure 1.

\section{Death During Surgery and Additional Surgery}

None of the patients died during surgery to treat SEA. A second surgery was required in $8 \%(\mathrm{n}=$ 12) of patients with a mean time between the first and second SEA surgery of 60 days. 


\section{Postoperative Pre-discharge AIS Score Model}

One aim of this study was to use patient characteristics to create a model that would aid physicians in predicting the outcome of treating SEA with surgery. The study data set contained 28 preoperative variables, therefore, a reduction process was applied and the final model only included variables where $\mathrm{R}^{2}$ changed by more than 0.01 . These variables are diabetes, high blood pressure, age, paresis, COPD, prior spinal surgery, osteomyelitis, preoperative pain duration due to SEA, and preoperative AIS score.

\section{Model Performance}

The concordance index provides a measure of a model's ability to discriminate among the levels of the outcome, here, the five levels of the predischarge AIS score. The concordance index is a value between 0.5 (coin flip) and 1.0 (perfect ability to rank patients). Jacknife concordance indices were calculated for predischarge AIS scores and resulted in $\mathrm{A}=0.98, \mathrm{~B}=0.96, \mathrm{C}=0.83$, $\mathrm{D}=0.84$ and $\mathrm{E}=0.91$. For example, $98 \%$ of the time, the predicted probability of a level $\mathrm{A}$ predischarge AIS score is higher for a patient who achieved a level A predischarge AIS score compared to a patient who has any otherlevel $(\mathrm{B}, \mathrm{C}, \mathrm{D}$, or E). The model displays good discrimination capability.

\section{Calibration of Model Predictions}

Leave-one-out cross-validation was used to explore the calibration of the model predictions by comparing the proportion of patients predicted to achieve each outcome level versus the actual proportion achieving that outcome level. Since each patient has a range of predicted probabilities, the predicted outcome level was taken as the maximum probability in the range. In general, if a patient is predicted to achieve a level $\mathrm{D}$ or $\mathrm{E}$, then the model is quite good at assigning the maximum probability to that level. The model, however, had more difficulty if a patient is predicted to achieve the lower levels $(\mathrm{A}, \mathrm{B}$, or $\mathrm{C})$ as there was less data available here. 


\section{Discussion}

SEA's are associated with significant risk of morbidity and mortality. ${ }^{9-11}$ To date, there are no comprehensive, evidence-based criteria for predicting the outcomes after treating SEA via operative management. Although several prior studies analyzed risk factors for poor outcomes in patients with SEA, no study used a multivariable model that includes all of the risk factors linked to poor perioperative outcomes. ${ }^{1,2,4,6-8}$ Compared to strictly medical management of SEA's, there are relatively few studies focusing exclusively on surgical and antibiotic management of SEA's. Therefore, the objectives of this study were to assess the clinical outcomes of SEA after surgical management, to identify patient-level factors that are associated with outcomes following surgical decompression of SEA, and to develop a predictive model for determining outcomes after surgical intervention. In the present study, the presence and duration of preoperative paresis were associated with remaining at the same or a worsening AIS score at the postoperative pre-discharge encounter $(\mathrm{p}<0.001)$ and a predictive model for post-surgical outcomes was developed.

\section{Patient Characteristics Associated with Pre-discharge AIS Scores}

In an analysis of 87 patients by Ghobrial et al., the only other study to utilize AIS scores as an outcome among SEA patients, the authors found a relative benefit of surgery within 24 hours of admission as measured by neurological grade at discharge. ${ }^{12}$ However, the authors did not analyze the relation of paresis to outcome despite finding that all patients who underwent surgery within 24 hours of admission had the same or improved AIS score at discharge. In their study of 30,274 surgically treated SEA patients, Schoenfeld and Wahlquist found that paralysis of any degree was associated with increased risk of morbidity, mortality, and increased cost of treatment. ${ }^{13}$ Importantly, the authors did not differentiate between partial and total paralysis. Sendi et al. reported in their review of medically and surgically treated SEA patients that if the duration of paresis is longer than 24-36 hours, neurological improvement is unlikely and that 
therefore, prolonged paresis is an indication for decompression surgery. ${ }^{7}$ Patel et al. in their study of 128 SEA patients found that neurologic outcomes can be improved through early surgical intervention rather than postponing surgery due to attempting medical treatment only. ${ }^{14}$ There is wide agreement that complete paralysis for more than 24-36 hours is associated with very poor outcomes and this characteristic is a relative contraindication to treating these patient's SEAs surgically. ${ }^{11,15-19}$

In the present study, both the presence and a mean duration of 1 day of preoperative paresis were associated with an increased risk of remaining at the same AIS score or worsening at the postoperative encounter (both $\mathrm{p}<0.001$ ). Our findings align with the general trend that delayed treatment can lead to poorer outcomes. The presence of paresis is also a factor in our predischarge AIS score prediction model which adds understanding on the surgical management of SEA.

In 82 patients 50 years and older, Adogwa et al. found that plegia was associated with poor long term outcomes. ${ }^{20}$ Similarly, Hlavin et al. reported that 0 out of 7 patients with plegia improved after operative management. ${ }^{17}$ Ghobrial et al. found that five out of six patients presenting with plegia remained at the same level of plegia at discharge. ${ }^{12}$ These studies are indicative of the broader surgical SEA literature: small sample sizes of operative patients leading to inconclusive results. The present study also has relatively few patients with plegia, which may limit our ability to draw any conclusions about the association between plegia and postoperative outcomes for surgical SEA patients.

\section{Pre-discharge and Follow-up AIS Scores}

Connor et al. found 43/55 (78\%) of surgically treated patients significantly improved or resolved at time of discharge. ${ }^{21}$ The remainder had no improvement, worsened, or died. Ghobrial et al.'s study found that $62 \%$ of patients maintained an AIS score of E or improved at discharge, $33 \%$ maintained the same score, and $5 \%$ worsened. ${ }^{12}$ The same study also showed $79 \%$ of surgical patients achieved an AIS score of E or D at the time of discharge, though due to small sample size and population variation no significant associations could be demonstrated. ${ }^{12}$ 
A comparison of the preoperative AIS and postoperative pre-discharge AIS scores in the present study showed that $49 \%$ of patients maintained an AIS score of $E$ or improved while $45 \%$ remained at their preoperative status and $6 \%$ worsened. At the same pre-discharge evaluation, $79 \%$ of patients achieved an E or D AIS score. Our data are similar to those reported by Ghobrial, though the results of the present study were worse than those in Connor et al. ${ }^{12,21}$ Our data suggest that many patients treated with surgery will likely achieve a score of E or improve and that a high percentage are likely to be free of or nearly free of weakness at the time of discharge.

Soehle and Wallenfang reported that $60 \%$ of patients treated surgically had good outcomes at a mean follow-up time of 11 months (good defined as slightly disabled but independently ambulatory or without neurological deficit). ${ }^{8}$ Patel et al. found that SEA patients managed operatively had an ASIA motor score increase of 9.52 points and a total score of 89.84 out of 100 at a mean follow-up time of 241.1 days. ${ }^{14}$ Khanna et al. reported $56.7 \%$ of surgical patients had a "good" outcome and $43.3 \%$ had a "poor" outcome measured at mean follow up time of 20.9 months (range, 4-45 months) (no definitions of "good" or "poor" outcomes were given, mean follow-up time was for medically and surgically treated patients in the study). ${ }^{4}$ In a study of patients over 50 years old and treated with surgery, Adogwa et al. reported 23\% with better outcomes, $33 \%$ unchanged, $37 \%$ worsened or died at mean follow up time of $41.38+/-86.48$ weeks (better defined as "neurologically better"). ${ }^{20}$

Among a subset of patients $(n=36 ; 23 \%)$ for whom a 6 month follow-up encounter occurred, $75 \%$ maintained an AIS score of E or improved, 19\% remained at their preoperative status, and $6 \%$ worsened. At the same encounter, $97 \%$ of patients achieved an E or D score. Comparison to other studies with respect to outcomes is made difficult due to variation in grading outcomes and time of follow-up measurement. Data on AIS scores at discharge and longer term follow-up may be useful in counseling patients on expectations of outcome when treating SEA with surgery. Therefore, further research that follows patients for longer periods postoperatively is warranted to develop better clinical decision-making and counseling tools. 
While death is a possible outcome of SEA, with a mortality rate of $1.8-25 \%$ cited in the literature, prior studies do not clarify at what stage in the natural history of the condition or treatment that death occurred. ${ }^{3}$ Additionally, to the authors' knowledge, no prior studies investigated the need for additional surgeries to treat SEA.

None of the 154 patients in the present study died in the course of treating SEA with surgery. In $8 \%$ of the patients in the present study, a second spinal surgery was required to treat the SEA. These data may be useful in counseling patients on the risk of surgery, potential for additional procedures, and aid in setting expectations.

\section{Postoperative Pre-discharge AIS Score Model}

There are several algorithms and grading schemes related to the treatment of SEA. Algorithms for the management of SEA were developed by Tuchman, Shweikeh, and Koppel, with a focus on selecting between medical vs. surgical management. ${ }^{1,22,23}$ Davis' decision guideline tool is centered on diagnosis of SEA in the Emergency Department. ${ }^{2}$ Khanna's grading scheme uses age, degree of thecal sac compression, and duration of symptoms to predict a good or poor outcome in either medical or surgical management. ${ }^{4}$ To the authors' knowledge, there are currently no models available, which enable the user to predict an AIS score for surgically managed SEA patients.

Our study data enabled the creation of an interactive model to predict the postoperative predischarge AIS score (http://riskcalc.org:3838/AIS_Score/). The model incorporates preoperative AIS score, pain duration, age, and status of osteomyelitis, preoperative spinal surgery, COPD, paresis, high blood pressure, and diabetes mellitus. Our model is a free webbased tool that enables the user to enter patient characteristics as outlined above. It calculates the probability that a patient will achieve each of the AIS scores at their pre-discharge evaluation. This new capability can be useful for the physician in understanding potential outcomes and in patient education. For example, if a patient currently has an AIS score of $\mathrm{E}$ and is unsure of their treatment choice, their physician can model the probabilities of their pre-discharge condition deteriorating to an AIS of C. Interestingly, patients with a history of COPD and/or high blood pressure have slightly improved probabilities of achieving better AIS scores. Other algorithms 
predicting the outcomes of SEA management have been developed, though focus on nonoperative management or 90-day mortality. ${ }^{24-26}$

\section{Limitations}

The retrospective design of our study limits it with the typical biases in data collection. The study is also not a comparative study comparing two different treatment methods and is therefore not randomized. Our 6 month follow-up data are limited by the lack of follow-up by patients for these encounters. Patients in this sub-set may have been more or less likely to follow-up based on their condition, which may have introduced selection bias in those results, particularly for patients whose outcomes were more positive neurologically. Allocation bias may also be a factor in that surgery may have been selected in cases where the patient's neurological condition was worse or imaging indicated a larger abscess.

\section{Conclusions}

Surgical decompression as part of the management of SEA can contribute to improving or maintaining AIS scores in a high percentage of patients. The presence and duration of preoperative paresis are prognostic for poorer outcomes and suggest that rapid surgical intervention before paresis develops may lead to improved postoperative outcomes. Our AIS score modeling tool enables physicians to enter patient characteristics and understand the probabilities of their pre-discharge condition. 


\section{References}

1. Tuchman A, Pham M, Hsieh PC. The indications and timing for operative management of spinal epidural abscess: literature review and treatment algorithm. Neurosurgical focus. 2014;37(2):E8.

2. Davis DP, Salazar A, Chan TC, Vilke GM. Prospective evaluation of a clinical decision guideline to diagnose spinal epidural abscess in patients who present to the emergency department with spine pain. Journal of Neurosurgery: Spine. 2011;14(6):765-770. doi:10.3171/2011.1.SPINE1091.

3. Epstein NE. Timing and prognosis of surgery for spinal epidural abscess: a review. Surgical neurology international. 2015;6(Suppl 19):S475.

4. Khanna RK, Malik GM, Rock JP, Rosenblum ML. Spinal epidural abscess: evaluation of factors influencing outcome. Neurosurgery 1996 39:958-964.

5. Reihsaus E, Waldbaur H, Seeling W. Spinal epidural abscess: a meta-analysis of 915 patients. Neurosurgical review. 2000;23(4):175-204.

6. Arko L, Quach E, Nguyen V, Chang D, Sukul V, Kim B-S. Medical and surgical management of spinal epidural abscess: a systematic review. Neurosurg Focus. 2014;37(2):E4, doi:10.3171/2014.6.FOCUS14127.

7. Sendi P, Bregenzer T, Zimmerli W. Spinal epidural abscess in clinical practice. QJM. 2007;101(1):1-12. doi:10.1093/qjmed/hcm100.

8. Soehle M, Wallenfang T. Spinal epidural abscesses: clinical manifestations, prognostic factors, and outcomes. Neurosurgery. 2002;51(1):79-87.

9. Marino RJ, Graves DE. Metric properties of the ASIA motor score: Subscales improve correlation with functional activities. Archives of Physical Medicine and Rehabilitation. 2004;85(11):1804-1810. doi:10.1016/j.apmr.2004.04.026. 
10. Schoenfeld AJ. Spine infections. In: Cannada L, ed. Orthopaedic Knowledge Update 11. Rosemont, IL: American Academy of Orthopaedic Surgeons, 2014:737-47.

11. Darouiche RO. Spinal epidural abscess. New England Journal of Medicine. 2006;355(19):2012-2020.

12. Ghobrial GM, Beygi S, Viereck MJ, et al. Timing in the surgical evacuation of spinal epidural abscesses. Neurosurgical focus. 2014;37(2):E1.

13. Schoenfeld AJ, Wahlquist TC. Mortality, complication risk, and total charges after the treatment of epidural abscess. The Spine Journal. 2015;15(2):249-255. doi:10.1016/j.spinee.2014.09.003.

14.Patel AR, Alton TB, Bransford RJ, Lee MJ, Bellabarba CB, Chapman JR. Spinal epidural abscesses: risk factors, medical versus surgical management, a retrospective review of 128 cases. The Spine Journal. 2014;14(2):326-330. doi:10.1016/j.spinee.2013.10.046.

15.Danner RL, Hartman BJ. Update of Spinal Epidural Abscess: 35 Cases and Review of the Literature. Clinical Infectious Diseases. 1987;9(2):265-274. doi:10.1093/clinids/9.2.265.

16. Darouiche RO, Hamill RJ, Greenberg SB, Weathers SW, Musher DM. Bacterial spinal epidural abscess: review of 43 cases and literature survey. Medicine. 1992;71(6):369-385.

17. Hlavin ML, Kaminski HJ, Ross JS, Ganz E. Spinal Epidural Abscess: A Ten-Year Perspective. Neurosurgery. 1990;27(2):177-184. doi:10.1227/00006123-19900800000001.

18. Rigamonti D, Liem L, Sampath P, et al. Spinal epidural abscess: contemporary trends in etiology, evaluation, and management. Surgical neurology. 1999;52(2):189-197.

19. Yang SY: Spinal epidural abscess. N Z Med J. 1982; 95:302-304. 
20. Adogwa O, Karikari IO, Carr KR, et al. Spontaneous spinal epidural abscess in patients 50 years of age and older: a 15-year institutional perspective and review of the literature. Journal of Neurosurgery: Spine. 2014;20(3):344-349.

21. Connor Jr DE, Chittiboina P, Caldito G, Nanda A. Comparison of operative and nonoperative management of spinal epidural abscess: a retrospective review of clinical and laboratory predictors of neurological outcome. Journal of Neurosurgery: Spine. 2013;19(1):119-127.

22. Shweikeh F, Saeed K, Bukavina L, Zyck S, Drazin D, Steinmetz MP. An institutional series and contemporary review of bacterial spinal epidural abscess: current status and future directions. Neurosurgical focus. 2014;37(2):E9.

23. Koppel BS, Tuchman AJ, Mangiardi JR, Daras M, Weitzner I. Epidural spinal infection in intravenous drug abusers. Archives of neurology. 1988;45(12):13311337.

24. Shah AA, Ogink PT, Nelson SB, Harris MB, Schwab JH. Nonoperative Management of Spinal Epidural Abscess: Development of a Predictive Algorithm for Failure. The Journal of Bone and Joint Surgery. 2018;100(7):546-555.

25. Kim SD, Melikian R, Ju KL, et al. Independent predictors of failure of nonoperative management of spinal epidural abscesses. The Spine Journal. 2014;14(8):1673-1679.

26. Shah AA, Ogink PT, Harris MB, Schwab JH. Development of Predictive Algorithms for Pre-Treatment Motor Deficit and 90-Day Mortality in Spinal Epidural Abscess: The Journal of Bone and Joint Surgery. 2018;100(12):1030-1038. 


\section{Figure Captions}

Figure 1. Preoperative and Postoperative AIS Scores

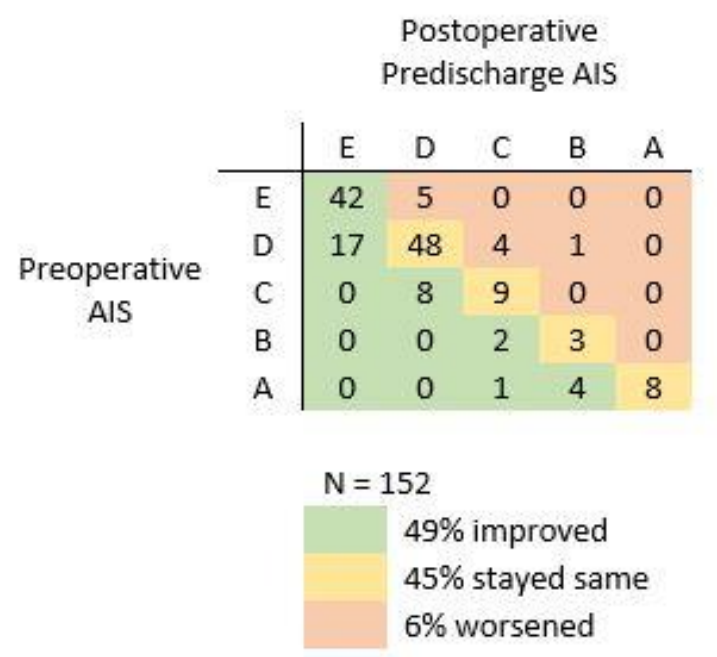

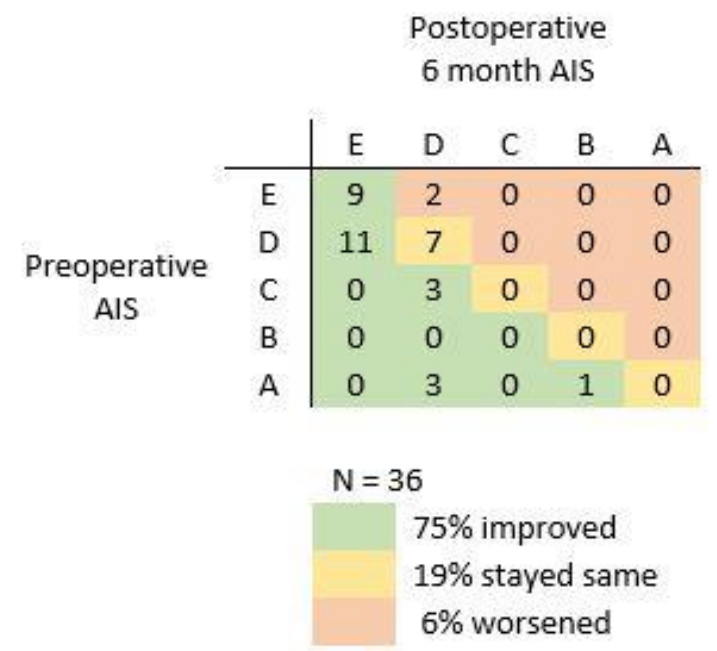

Table 1. Characteristics of Spinal Epidural Abscess Surgery Patients

Patient Characteristics

$\mathbf{N}=154$

\begin{tabular}{lc}
\hline Age, years & $58.0 \pm 12.8$ \\
White & $125(81 \%)$ \\
Male & $94(61 \%)$ \\
Body Mass Index ${ }^{\mathrm{a}}, \mathrm{kg} / \mathrm{m}^{2}$ & $28.2(24.4-33.4)$ \\
Current Smoker & $35(24 \%)$ \\
$\begin{array}{l}\text { Admission Source } \\
\text { non-facility point of origin }\end{array}$ & $44(29 \%)$ \\
$\quad$ transfer from a different hospital/facility & $96(62 \%)$ \\
$\quad$ clinic referral & $12(8 \%)$ \\
$\quad$ emergency room & $2(1 \%)$
\end{tabular}


Radiculopathy

$53(34 \%)$

Neck/Back Pain

$123(80 \%)$

Duration of Neck/Back Pain, days

$7(2-19)$

Bladder/Bowel Dysfunction

$34(22 \%)$

Paresis

$73(47 \%)$

Duration of Weakness, days

$0(0-5)$

Plegia

$24(16 \%)$

Numbness

$33(21 \%)$

Duration of Numbness, days

$0(0-0)$

Paraspinal Abscess

$48(31 \%)$

Staphylococcus Aureus

$71(46 \%)$

C-reactive protein (CRP), mg/L

$121(52-240)$

White Blood Cells (WBC) Count, (k/microliter)

$10.9(8.2-14.7)$

Chronic Steroid Use

$12(8 \%)$

Bacteremia

81 (53\%)

MRSA

$25(17 \%)$

Osteomyelitis

76 (49\%)

Platelets, (k/microliter)

$294(214-380)$

Prior Spinal Surgery

$41(27 \%)$

IV Drug Use History

$13(8 \%)$

Anti-depressants

$40(27 \%)$

Chronic Renal Failure

$16(10 \%)$

Other Renal Complications

$17(11 \%)$

Hepatic Disease

$21(14 \%)$

Coronary Artery Disease

$21(14 \%)$

High Blood Pressure

89 (58\%)

Diabetes Mellitus

Type I

Type II

$46(30 \%)$ 
Congestive Heart Failure

Chronic Obstructive Pulmonary Disease

Erythrocyte Sedimentation Rate ${ }^{b}, \mathrm{~mm} / \mathrm{hr}$
$13(8 \%)$

$18(12 \%)$

$76.3 \pm 30.4$

\footnotetext{
${ }^{a}$ missing for 48 subjects

${ }^{\mathrm{b}}$ missing for 47 subjects

descriptive statistics are either count (\%), mean \pm standard deviation or median $\left(Q_{1}-Q_{3}\right)$
}

Table 2. Associations Among SEA Patient Characteristics and Surgical Outcomes

\begin{tabular}{|c|c|c|c|c|c|c|}
\hline \multirow[b]{2}{*}{ Patient Characteristics } & \multicolumn{2}{|c|}{ Postoperative Predischarge AIS } & \multirow[b]{2}{*}{$\mathbf{p}$} & \multicolumn{2}{|c|}{ Postoperative 6 months AIS } & \multirow[b]{2}{*}{ p } \\
\hline & Improve & Same/Worse & & Improve & Same/Worse & \\
\hline Count & 74 & 78 & & 27 & 9 & \\
\hline Age, years & $57.3 \pm 13.4$ & $58.7 \pm 12.4$ & 0.61 & $57.1 \pm 14.9$ & $53.3 \pm 8.7$ & 0.25 \\
\hline White & $85 \%$ & $78 \%$ & 0.30 & $89 \%$ & $78 \%$ & 0.58 \\
\hline Male & $62 \%$ & $60 \%$ & 0.87 & $52 \%$ & $78 \%$ & 0.25 \\
\hline Body Mass Index ${ }^{\mathrm{a}}, \mathrm{kg} / \mathrm{m}^{2}$ & $28.9(25.7-33.4)$ & $27.6(23.7-34.0)$ & 0.27 & $29.7(26.1-37.2)$ & $27.5(26.7-30.3)$ & 0.31 \\
\hline Current Smoker & $23 \%$ & $23 \%$ & 0.99 & $38 \%$ & $33 \%$ & 0.99 \\
\hline Radiculopathy & $38 \%$ & $31 \%$ & 0.40 & $30 \%$ & $33 \%$ & 0.99 \\
\hline Neck/Back Pain & $82 \%$ & $77 \%$ & 0.43 & $74 \%$ & $89 \%$ & 0.65 \\
\hline Duration of Neck/Back Pain, days & $8(3-16)$ & $7(2-21)$ & 0.81 & $8(4-21)$ & $7(4-15)$ & 0.98 \\
\hline Bladder/Bowel Dysfunction & $23 \%$ & $22 \%$ & 0.99 & $15 \%$ & $22 \%$ & 0.63 \\
\hline Paresis & $32 \%$ & $63 \%$ & 0.0002 & $59 \%$ & $56 \%$ & 0.99 \\
\hline Duration of Paresis, days & $0(0-1)$ & $1(0-7)$ & 0.0003 & $0(0-6)$ & $1(0-1)$ & 0.72 \\
\hline Plegia & $11 \%$ & $21 \%$ & 0.12 & $15 \%$ & $22 \%$ & 0.63 \\
\hline Numbness & $20 \%$ & $23 \%$ & 0.70 & $22 \%$ & $33 \%$ & 0.66 \\
\hline Duration of Numbness, days & $0(0-0)$ & $0(0-0)$ & 0.69 & $0(0-0)$ & $0(0-1)$ & 0.79 \\
\hline Paraspinal Abscess & $34 \%$ & $29 \%$ & 0.60 & $7 \%$ & $44 \%$ & 0.02 \\
\hline Staphylococcus Aureus & $43 \%$ & $49 \%$ & 0.52 & $52 \%$ & $67 \%$ & 0.70 \\
\hline C-reactive protein (CRP), $\mathrm{mg} / \mathrm{L}$ & $133(49-239)$ & $93(53-241)$ & 0.74 & $115(62-194)$ & $312(246-427)$ & 0.049 \\
\hline White Blood Cells (WBC) Count, (k/microliter) & $10.6(7.9-13.7)$ & $11.2(8.4-15.3)$ & 0.22 & $12.2(8.5-17.8)$ & $15.3(12.9-18.7)$ & 0.46 \\
\hline Chronic Steroid Use & $8 \%$ & $8 \%$ & 0.99 & $8 \%$ & $0 \%$ & 0.99 \\
\hline Bacteremia & $52 \%$ & $55 \%$ & 0.75 & $52 \%$ & $44 \%$ & 0.99 \\
\hline
\end{tabular}




\begin{tabular}{|c|c|c|c|c|c|c|}
\hline MRSA & $14 \%$ & $19 \%$ & 0.39 & $11 \%$ & $0 \%$ & 0.56 \\
\hline Osteomyelitis & $43 \%$ & $55 \%$ & 0.15 & $44 \%$ & $67 \%$ & 0.44 \\
\hline Platelets, (k/microliter) & $258(214-385)$ & $307(212-377)$ & 0.44 & $276(202-331)$ & 390 (294 - 459) & 0.049 \\
\hline Prior Spinal Surgery & $22 \%$ & $31 \%$ & 0.27 & $30 \%$ & $11 \%$ & 0.40 \\
\hline IV Drug Use History & $9 \%$ & $8 \%$ & 0.78 & $11 \%$ & $11 \%$ & 0.99 \\
\hline Anti-depressants & $33 \%$ & $21 \%$ & 0.13 & $16 \%$ & $0 \%$ & 0.55 \\
\hline Chronic Renal Failure & $7 \%$ & $14 \%$ & 0.19 & $11 \%$ & $0 \%$ & 0.56 \\
\hline Other Renal Complications & $11 \%$ & $12 \%$ & 0.99 & $4 \%$ & $11 \%$ & 0.44 \\
\hline Hepatic Disease & $14 \%$ & $14 \%$ & 0.99 & $4 \%$ & $0 \%$ & 0.99 \\
\hline Coronary Artery Disease & $12 \%$ & $14 \%$ & 0.81 & $7 \%$ & $11 \%$ & 0.99 \\
\hline High Blood Pressure & $57 \%$ & $58 \%$ & 0.99 & $52 \%$ & $22 \%$ & 0.24 \\
\hline Diabetes Mellitus & & & 0.61 & & & 0.37 \\
\hline Type I & $1 \%$ & $3 \%$ & & $0 \%$ & $11 \%$ & \\
\hline Type II & $27 \%$ & $32 \%$ & & $22 \%$ & $22 \%$ & \\
\hline Congestive Heart Failure & $5 \%$ & $12 \%$ & 0.25 & $0 \%$ & $11 \%$ & 0.25 \\
\hline Chronic Obstructive Pulmonary Disease & $15 \%$ & $6 \%$ & 0.11 & $15 \%$ & $0 \%$ & 0.55 \\
\hline Erythrocyte Sedimentation Rate ${ }^{\mathrm{b}}, \mathrm{mm} / \mathrm{hr}$ & $74.1 \pm 31.3$ & $78.7 \pm 29.6$ & 0.31 & $77 \pm 37$ & $96 \pm 13$ & 0.23 \\
\hline
\end{tabular}

descriptive statistics are either count $(\%)$, mean \pm standard deviation or median $\left(Q_{1}-Q_{3}\right)$

p-values result from either Fisher's exact (categorical) or Wilcoxon rank-sum (continuous) tests

AIS = American Spinal Injury Association Impairment Scale scores

Table 3. Characteristics of Spinal Epidural Abscesses

\section{SEA Characteristics}

Region of Abscess

cervical

cervico-thoraco-lumbar

cervico-thoraco-lumbo-sacral

cervicothoracic

lumbar
$\mathbf{N}=154$

$33(21 \%)$

$1(1 \%)$

$1(1 \%)$

$7(4 \%)$

$44(28 \%)$ 
lumbosacral

thoracic

thoraco-lumbo-sacral

thoracolumbar

Abscess Location

dorsal

dorsolateral left

dorsolateral right

left

right

ventral

ventrolateral left

ventrolateral right

other

Degree of Compression

mild to moderate

severe

unable to read

no $\mathrm{MRI} / \mathrm{CT}$
$17(11 \%)$

$41(27 \%)$

$4(3 \%)$

$6(4 \%)$

$63(41 \%)$

$4(3 \%)$

$3(2 \%)$

$4(3 \%)$

$6(4 \%)$

$56(36 \%)$

$2(1 \%)$

$6(4 \%)$

$10(6 \%)$

$52(34 \%)$

$89(58 \%)$

$3(2 \%)$

$10(6 \%)$

Table 4. Associations Among Abscess Characteristics and Surgical Outcomes

\section{Postoperative \\ Predischarge AIS \\ Postoperative \\ 6 months AIS}

Patient Characteristics

$$
\text { Improve Same/Worse }
$$

p Improve Same/Worse p
Count

Region of Abscess

cervical
63

0.17
21

$20 \%$

$35 \%$

$33 \%$

$40 \%$ 
lumbar

thoracic

Count

Abscess Location

ventral

dorsal

Count

Degree of Compression

mild to moderate

severe
$44 \%$

$35 \%$

43

46

0.13

$33 \%$

$67 \%$

69

71

$50 \%$

$38 \%$

$62 \%$

24

9

0.39

$41 \%$

$67 \%$

$11 \%$

$67 \%$

$59 \%$

$33 \%$

$89 \%$

p-values result from Fisher's exact tests

AIS = American Spinal Injury Association Impairment Scale scores

Table 5. Postoperative AIS Scores

\begin{tabular}{|c|c|c|c|}
\hline \multicolumn{4}{|c|}{ Postoperative Predischarge AIS } \\
\hline improvement & $74(49 \%)$ & achieved E/D status & $120(79 \%)$ \\
\hline stayed same & $68(45 \%)$ & achieved $\mathrm{C} / \mathrm{B} / \mathrm{A}$ status & $32(21 \%)$ \\
\hline worsened & $10(6 \%)$ & & \\
\hline
\end{tabular}

\begin{tabular}{|c|c|c|c|}
\hline \multicolumn{4}{|c|}{ Postoperative 6 month AIS } \\
\hline improvement & $27(75 \%)$ & achieved E/D status & $35(97 \%)$ \\
\hline stayed same & 7 (19\%) & achieved $\mathrm{C} / \mathrm{B} / \mathrm{A}$ status & $1(3 \%)$ \\
\hline worsened & $2(6 \%)$ & & \\
\hline
\end{tabular}

AIS = American Spinal Injury Association Impairment Scale scores 
Table 6. Classification Table of Predicted and Actual Postoperative, Predischarge AIS Score

\begin{tabular}{|c|c|c|c|c|c|c|c|}
\hline & \multicolumn{5}{|c|}{ Actual Postoperative Predischarge AIS Score Level } & \multirow[b]{2}{*}{ Total } \\
\hline & & A & B & C & D & $E$ & \\
\hline \multirow{5}{*}{$\begin{array}{c}\text { Predicted } \\
\text { Postoperative } \\
\text { Predischarge AIS } \\
\text { Score Level }\end{array}$} & A & $4(44 \%)$ & $5(56 \%)$ & $0(0 \%)$ & $0(0 \%)$ & $0(0 \%)$ & $9(6 \%)$ \\
\hline & B & $2(29 \%)$ & $2(29 \%)$ & $3(43 \%)$ & $0(0 \%)$ & $0(0 \%)$ & $7(5 \%)$ \\
\hline & C & $0(0 \%)$ & $0(0 \%)$ & $4(50 \%)$ & $4(50 \%)$ & & $8(5 \%)$ \\
\hline & D & $0(0 \%)$ & $0(0 \%)$ & $8(11 \%)$ & $50(70 \%)$ & $101+0$ & $71(49 \%)$ \\
\hline & $\mathbf{E}$ & $0(0 \%)$ & $0(0 \%)$ & $1(2 \%)$ & $6(12 \%)$ & 1800 & $51(35 \%)$ \\
\hline & Total & $6(4 \%)$ & $7(5 \%)$ & $16(11 \%)$ & $60(41 \%)$ & $57(39 \%)$ & 146 \\
\hline
\end{tabular}

